\title{
Treatment of AC-joint instability: what seems to be a small thing still leaves us with numerous open questions
}

\author{
Klaus Bak ${ }^{1}$ (1) Knut Beitzel ${ }^{2}$
}

Received: 26 June 2019 / Accepted: 5 August 2019 / Published online: 19 August 2019

(c) European Society of Sports Traumatology, Knee Surgery, Arthroscopy (ESSKA) 2019

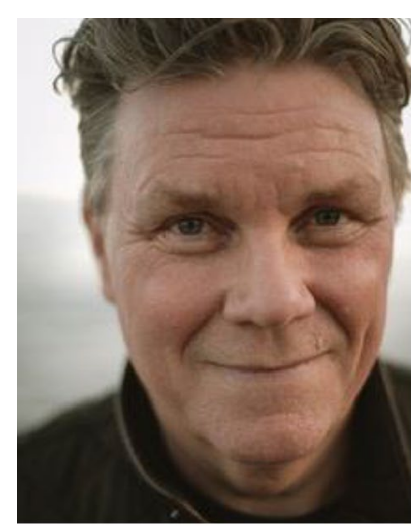

Klaus Bak

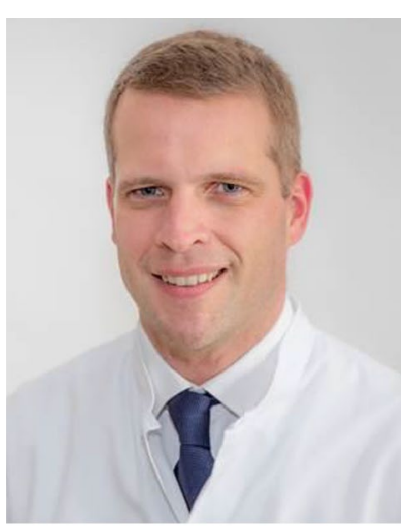

Knut Beitzel
We are proud to publish a handful of selected high-quality papers dealing with acromioclavicular instability in this issue. The acromioclavicular (AC) joint is a relatively small but essential joint that has an important effect on shoulder function. Dislocation of the AC-joint is the most common acute shoulder injury in sports, and treatment of this injury remains controversial worldwide.

Despite an increasing number of studies, there are still many diatribes both with regard to diagnosis, classification, best initial treatment and universally accepted and validated injury-specific outcome scores. We have to be aware that our classic scores for clinical outcome evaluation might not entirely capture the patient's subjective problems with regard to AC-joint instability. Therefore, we might miss clinically relevant differences in our studies. Another factor is finding the correct indication for each treatment. Imaging has

Klaus Bak

skulderbak@gmail.com

1 Kysthospitalet Skodsborg, Skodsborg Strandvej 125A, 3rd, 2942 Skodsborg, Denmark

2 ATOS Clinic Cologne, Aachener Str. 1021B, 50858 Cologne, Germany significant limitations in clearly defining the diagnosis and severity of the injury. In the future, we need to have a more functional approach to finding our indication for surgical therapy rather than just the imaging classification. Perhaps we need more patient-specific criteria to better find the candidates, who can profit from surgical treatment.

Numerous authors have published surgical procedures to deal with this injury. During the last decade, there have been increased focus and further science supporting more precise anatomic and less invasive repair methods aiming at recreating rotational, horizontal and vertical stability. However, there are still only a few randomized studies comparing operative and non-operative treatment, and these show that operative treatment is overall not in favour of conservative treatment in a general population. In addition, the surgical methods used in these studies are mainly non-anatomic. Until 2015, the latest randomized controlled trial (RCT) was dated 1986.

There is no doubt that we are getting closer in identifying the right treatment for this injury, but the road ahead is still long. The vast majority of papers published and submitted are small uncontrolled series on different operative techniques with short follow-ups. Also, despite a great improvement in techniques, the results are still imperfect with frequent complications reported. On the other hand, nonoperative treatment and methods are only sparsely reported in the literature, and to date no prospective cohort studies or controlled studies evaluating non-operative treatment have been published. As with other injuries subjected to planned RCTs, the AC-injured may need more individualized treatment, or at least the indication for surgery or conservative treatment may vary from case to case. This makes it difficult to collect a sufficient number of patients for an RCT within a predictable number of years. For this reason, you may end up with a well-designed study with a few limitations resulting in a general conclusion that only fits the average individual. Additionally, we encounter patients with initial injuries and patients with failed treatment in our daily practice. In the 
face of low levels of evidence for the index surgery, it is even harder to find strategies to deal with surgical failures. The good news is that more and more high-quality biomechanical papers are being published which help us in understanding the complexity of the AC-joint biomechanics and function.

Finally, we find it important to publish both excellent results and unexpected bad results. Since we are still only at the threshold of solving the riddle, it is important to manage critical data in order to move forward so that we are able to discuss treatment options and offer the best to our patients. We hope that these carefully selected papers for this AC-theme issue will pique your interest and provide inspiration to design new studies to help us get closer to better solutions for the injured AC-joint.

Publisher's Note Springer Nature remains neutral with regard to jurisdictional claims in published maps and institutional affiliations. 